\title{
Envelhecimento, estresse oxidativo e sarcopenia: uma abordagem sistêmica
}

\section{Aging oxidativestressandsarcoperia asystemicapproach}

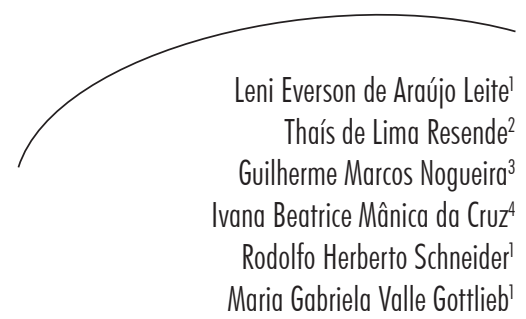

\section{Resumo}

Introdução: a sarcopenia é caracterizada pela perda de massa e funcionalidade muscular e está associada a uma série de disfunções e doenças sistêmicas que acometem os idosos. Também é um dos parâmetros utilizados para definição da síndrome de fragilidade, que é altamente prevalente em idosos, conferindo maior risco para quedas, fraturas, incapacidade, dependência, hospitalização recorrente e mortalidade. Recentemente, a literatura tem reportado que a etiopatogenia da sarcopenia está intimamente relacionada com um aumento na taxa de produção de espécie reativa de oxigênio (ERO), o que pode ocasionar a denervação, perda e atrofia de fibras musculares e consequente perda da força muscular. Objetivo: realizar uma revisão narrativa da literatura sobre o papel do estresse oxidativo na gênese da sarcopenia. Método: revisão narrativa. Foram consultadas as bases de dados MEDLINE, LILACS e SciElo. As buscas foram feitas através das expressões: sarcopenia e envelhecimento, perda de massa muscular em idosos, estresse oxidativo, inflamação e sarcopenia e epidemiologia da sarcopenia. Resultados: a etiologia da sarcopenia é multifatorial e envolve a interação de diversos fatores, incluindo aspectos genéticos, metabólicos, estilo de vida, como os hábitos alimentares (ingestão protéica) e de gasto energético (atividade física), e as EROs desempenham papel-chave na mediação do processo de perda de massa e função muscular associado ao envelhecimento. Conclusão: ao longo do processo de envelhecimento, o estresse oxidativo torna-se mais perigoso, uma vez que, associado ao declínio dos hormônios sexuais, que exercem efeitos anabólicos sobre o tecido músculo-esquelético, pode acelerar a perda e atrofia desse tecido.

Programa de Pós-Graduação em Gerontologia Biomédica, Instituto de Geriatria e Gerontologia. Pontifícia Universidade Católica do Rio Grande do Sul. Porto Alegre, RS, Brasil.

2 Faculdade de Enfermagem, Nutrição e Fisioterapia. Pontifícia Universidade Católica do Rio Grande do Sul. Porto Alegre, RS, Brasil.

3 Faculdade de Educação Física e Ciência do Desporto. Pontifícia Universidade Católica do Rio Grande do Sul. Porto Alegre, RS, Brasil.

4 Programa de Pós-Graduação em Bioquímica Toxicológica e Farmacologia, Departamento de Ciências da Saúde. Universidade Federal de Santa Maria. Santa Maria, RS, Brasil.

Correspondência / Correspondence

Maria Gabriela Valle Gottlieb

Pontifícia Universidade Católica do Rio Grande do Sul - PUCRS

Av. Ipiranga, 6690 - Hospital São Lucas - $3^{\circ}$ andar

90610-000 Porto Alegre-RS/Brasil

E-mail: maria.gottlieb@pucrs.br

Palavras-chave: Sarcopenia. Sistema Musculoesquelético. Metabolismo. Inflamação. Envelhecimento. Idoso. 


\section{Abstract}

Introduction: sarcopenia is characterized by loss of muscle mass and its function and is associated with a number of systemic diseases and disorders that affect the elderly. It is also one of the parameters used to define the frailty syndrome, which is highly prevalent among the elderly, conferring upon them greater risk for falls, fractures, disability, dependency, recurrent hospitalization and mortality. Recently there have been reports in the literature showing that the pathogenesis of sarcopenia is closely related to the breakdown of oxidative metabolism's homeostasis, leading to an increased rate of production of reactive oxygen species (ROS), causing mitochondrial damage, altering the immune and inflammatory system, resulting in muscle fibers' denervation, loss and atrophy and ensuing loss of muscle strength. Objective: to perform a narrative review on the role of oxidative stress in the etiology of sarcopenia. Method: narrative review. Were searched databases like MEDLINE, LILACS and Scielo. The searches were made through the expressions: sarcopenia and ageing, elderly muscular mass loss, oxidative stress, inflammation and sarcopenia and epidemiology and sarcopenia. Results: the etiology of sarcopenia is multifactorial and involves interaction of several factors, including genetic, metabolic and life style factors, such as eating habits (protein intake) and energy expenditure (physical activity), and the EROs play a key role in mediating the process of mass and muscle function loss associated with ageing. Conclusion: over the course of the ageing process the oxidative stress becomes more dangerous, since, associated with the decline of sexual hormones and life style which exert anabolic effects on the musculoskeletal tissue, may accelerate it the loss and atrophy.
Key words: Sarcopenia. Musculoskeletal System. Metabolism. Inflammation. Aging.

\section{INTRODUÇÃO}

O envelhecimento é um processo dinâmico, no qual ocorrem modificações morfológicas e fisiológicas em todos os níveis do organismo. Essas mudanças têm início relativamente cedo; alguns autores postulam que as mesmas acontecem ao final da segunda década da vida, perdurando longo tempo e sendo pouco perceptíveis até que surjam, ao final da terceira década, as primeiras alterações funcionais e/ou estruturais atribuídas ao envelhecimento. Tal processo leva a um progressivo decréscimo na capacidade fisiológica e redução da capacidade de respostas ao estresse ambiental, levando a um aumento da suscetibilidade e vulnerabilidade a doenças. ${ }^{1}$

Em uma revisão sobre biologia do envelhecimento, Troen ${ }^{1}$ descreveu que existiriam dois tipos de envelhecimento: o biológico normal e o usual. $\mathrm{O}$ envelhecimento normal envolve as mudanças biológicas inexoráveis e universais, características do processo. Já no envelhecimento usual, além das alterações biológicas, observa-se o aumento da incidência de doenças crônicas. ${ }^{1}$
Estas doenças se originam do acúmulo de danos, ao longo da vida, oriundos sobretudo da interação entre fatores genéticos e hábitos não saudáveis, como dieta desbalanceada, tabagismo, etilismo e sedentarismo. ${ }^{1}$ Um estilo de vida inapropriado acaba aumentando a ineficiência metabólica, que contribui substancialmente para a quebra da homeostasia corporal. Tal fato, lentamente, torna o indivíduo mais suscetível a lesões orgânicas, culminando no desencadeamento de doenças associadas ao processo de envelhecimento. Essas doenças são chamadas de crônicas nãotransmissíveis (DCNT) e podem desencadear processosincapacitantes, afetando afuncionalidade dos indivíduos idosos, dificultando ou impedindo o desempenho de suas atividades cotidianas de forma independente, o que pode comprometer de maneira significativa a qualidade de vida dos idosos, como é o caso da sarcopenia. ${ }^{2}$

O termo sarcopenia deriva do grego e significa "pobreza de carne"." A sarcopenia, que é caracterizada pela perda de massa muscular, está associada a uma série de disfunções e doenças sistêmicas prevalentes no idoso, como é o caso da 
osteoporose, ${ }^{4}$ resistência à insulina, ${ }^{5}$ obesidade ${ }^{6}$ e osteoartrite, ${ }^{7}$ além de causar complicações com o avanço da idade. O potencial impacto da sarcopenia é grande, considerando que o tecido muscular é o mais abundante do corpo humano. ${ }^{3,8}$

Segundo revisão de Silva et al.,

sarcopenia é uma das variáveis utilizadas para definição da sindrome de fragilidade, que é altamente prevalente em idosos, conferindo maior risco para quedas, fraturas, incapacidade, dependência, hospitalização recorrente e mortalidade.

A síndrome representa uma vulnerabilidade fisiológica relacionada à idade, resultado da deterioração da homeostase biológica e da capacidade do organismo de se adaptar às novas situações de estresse. ' Entretanto, o caráter reversível da sarcopenia é consenso entre a maioria dos especialistas, ${ }^{1,3,6,10}$ visto que está diretamente relacionada ao desempenho músculo-esquelético, que tem potencial para reabilitação com consequente restauração da capacidade física. Isto é, a atividade física desempenha papel fundamental na manutenção ou lentificação da perda de massa muscular. Dessa forma, o sedentarismo parece ser um fator de risco para a sarcopenia em idosos. ${ }^{11}$

Recentemente, evidências têm sugerido que o estresse oxidativo excessivo, ${ }^{12}$ disfunção mitocondrial ${ }^{13}$ e inflamação crônica ao longo do envelhecimento ${ }^{14}$ estão envolvidos em um subsequente declínio da força e mobilidade do idoso, que são considerados, entre outros, critérios diagnósticos para a sarcopenia. ${ }^{15}$ As evidências científicas têm sugerido que a bioenergética e função mitocondrial, via geração de espécias reativas de oxigênio (ERO), podem ter papel relevante no processo de degeneração neuromuscular, com consequente perda de fibras e função muscular associadas ao processo de envelhecimento. ${ }^{12,13,15}$ Desta forma, a produção de ERO ao longo do envelhecimento parece estar envolvida na patogênese da sarcopenia. ${ }^{15}$ Entretanto, ainda são pouco compreendidos os fatores e mecanismos interconectados que levam ao estresse oxidativo, e qual o reflexo dessa quebra de homeostasia redox celular no processo sarcopênico humano, dentro de uma abordagem sistêmica. Isto porque o estresse oxidativo atua no organismo de forma sistêmica, gerando uma cascata de eventos que podem desencadear diferentes DCNT interligadas.

Esse tipo de abordagem é relevante, uma vez que cada vez mais os estudos têm mostrado que a sarcopenia tem etiologia multifatorial, e que tanto a prevenção quanto o tratamento devem estar apoiados em fundamentos da interdisciplinaridade. Ou seja, para se compreender realmente o que promove o desencadeamento do processo sarcopênico, é fundamental que as diferentes áreas do saber construam uma interface. Dentro desse contexto, o presente artigo apresentará uma revisão sobre o papel do estresse oxidativo na gênese da sarcopenia.

\section{MÉTODO}

Este estudo é uma revisão narrativa, para a qual foram consultadas as bases de dados MEDLINE, LILACS e SciElo. As buscas foram feitas a partir do ano 1990, nas línguas português, espanhol e inglês, através das expressões: sarcopenia e envelhecimento, perda de massa muscular em idosos, estresse oxidativo, inflamação e sarcopenia e epidemiologia da sarcopenia. Os tipos de publicação utilizados foram ensaios clínicos, estudos experimentais, revisões e consensos.

Como critérios de inclusão, foram utilizados artigos cuja temática abordasse o envelhecimento e suas consequências no sistema músculoesquelético, metabolismo músculo-esquelético e celular, estresse oxidativo e inflamação, fatores físicos ambientais domésticos, comportamentais e quedas associados à perda de massa muscular em idosos, além de manuscritos que abordassem a prevalência de sarcopenia no mundo.

Em relação ao conhecimento sobre a estrutura e função do tecido muscular, utilizou-se como referência livro-texto considerado fonte de consulta básica para o entendimento do assunto. 


\section{REVISÃO DA LITERATURA}

O entendimento do processo pelo qual o estresse oxidativo pode levar à sarcopenia associada ao envelhecimento demanda clareza sobre a estrutura, a função e o funcionamento do tecido muscular humano, bem como as modificações decorrentes da senescência que o mesmo sofre. Assim sendo, a revisão da literatura se inicia por uma breve descrição desses aspectos, para depois abordar em sequência o envelhecimento músculo-esquelético e a sarcopenia, a epidemiologia da sarcopenia e, por fim, o papel do estresse oxidativo e da inflamação na etiopatogenia da sarcopenia.

\section{Tecido muscular: estrutura, função e funcionamento}

A massa corporal total humana é composta por aproximadamente $40 \%$ de massa magra ou massa livre de gordura (músculo esquelético), sendo que um quarto de toda a síntese protéica corporal ocorre no músculo. ${ }^{16}$ Grande parte do músculo esquelético está unida aos ossos do esqueleto, o que permite controlar os movimentos corporais. ${ }^{17}$ Esse músculos, assim como o cardíaco, é classificado como estriado (stria, sulco) em função de suas bandas claras e escuras alternadas (figura 1), que podem ser visualizadas em microscopia óptica. Os músculos esqueléticos são formados por um conjunto de células musculares ou fibras musculares, onde cada fibra é uma longa célula cilíndrica com até milhares de núcleos próximos da sua superfície. Cada fibra esquelética é envolta em tecido conectivo, com grupos de fibras adjacentes formando o fascículo. ${ }^{17}$

O tecido muscular esquelético (contração voluntária) é constituído por tipos diferentes de fibras com base na sua velocidade de contração e sua resistência à fadiga, decorrente de estimulação repetida: as fibras de contração lenta (ST ou tipo I), inervadas por pequenos neurônios motores alfa, as fibras glicolítico-oxidativas de contração rápida (FOG ou tipo IIA) e as glicolíticas de contração rápida (FG ou tipo IIB), inervadas por grandes neurônios motores. As fibras do tipo II desenvolvem a tensão duas vezes mais rápido do que as do tipo I e a velocidade com que a fibra contrai é determinada pela isoforma da ATPase da miosina dos filamentos grossos. As fibras glicolítico-oxidativas utilizam uma combinação do metabolismo oxidativo (fosforilação oxidativa) e glicolítico para a produção de trifosfato de adenosina (ATP), são mais resistentes à fadiga do que as fibras somente glicolíticas e apresentam grande quantidade de mioglobina, assim como as fibras de contração lenta. ${ }^{17}$ 


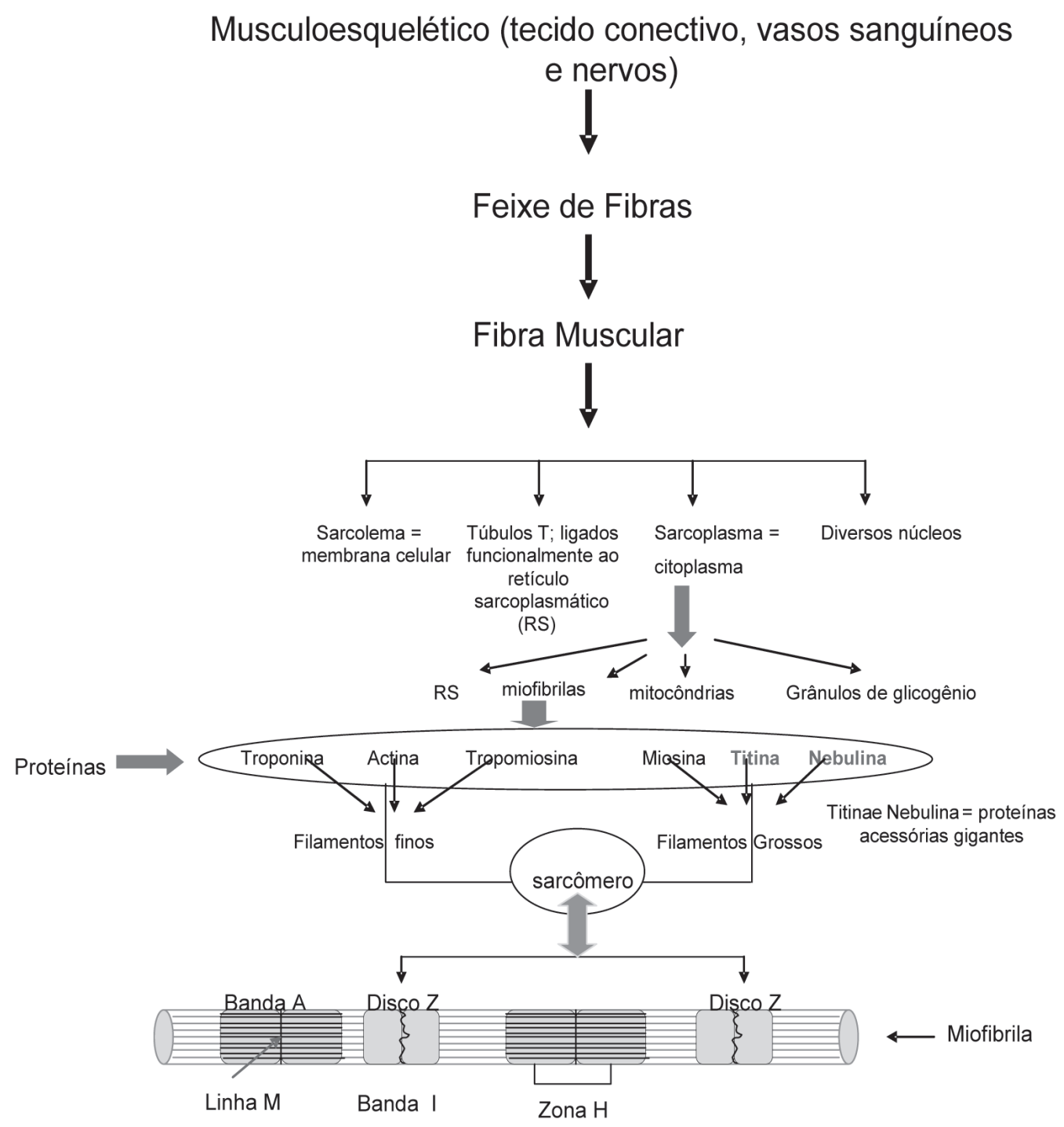

Figura 1. Esquema da Anatomia e ultraestrutura do músculo esquelético

Fonte: Silverthorn, 2010. ${ }^{17}$

Legenda: Banda A: mais escura das bandas; os filamentos grossos e finos se sobrepõem; Linha M: divide a banda A ao meio; representa as proteínas que formam o sítio de apoio para os filamentos grossos; Disco Z: estruturas proteicas em ziguezague que atuam como sítios de fixação para os filamentos finos. Um sarcômero é formado por dois discos Z e pelos filamentos situados entre eles; Banda I: banda mais clara do sarcômero e é ocupada somente pelos filamentos finos; Zona $\mathbf{H}$ : região central da banda A, ocupada somente por filamentos grossos. 


\section{Envelhecimento músculo-esquelético e sarcopenia}

O músculo esquelético é um tecido com alta susceptibilidade ao processo de envelhecimento, afetado por alterações progressivas e deletérias associadas à desorganização estrutural e funcional inerente à senescência. ${ }^{18}$ Segundo alguns autores, entre os 25 e 65 anos de idade há uma diminuição substancial da massa magra, da ordem de 10 a 16\%. ${ }^{11,18}$ Essa perda não se dá de forma uniforme entre os diferentes tipos de fibra que compõem o tecido muscular humano. Parece que, até os 70 anos de idade, as fibras do tipo I são mais resistentes à atrofia, enquanto que a área relativa às fibras do tipo II declina de 20 a $50 \%$ com o avanço da idade. ${ }^{19}$ Estudos com cadáveres descreveram uma redução de $50 \%$ do número de fibras musculares após a nona década de vida, quando comparados com indivíduos jovens (20 anos de idade). ${ }^{19}$

Durante o envelhecimento, a perda progressiva da massa muscular esquelética, com diminuição no número e tamanho das fibras tipo II e uma diminuição paralela da força e resistência muscular, são achados frequentes em diferentes populações. ${ }^{4,6,10,11,20}$ Esta condição, denominada sarcopenia, tem importantes implicações para a saúde e condições socioeconômicas dos idosos, uma vez que contribui para a fragilidade, perda funcional, dependência, deficiência, bem como aumenta os custos dos cuidados de saúde e pode levar à morte prematura. ${ }^{21}$ Tal processo ocorre, entre outras causas, devido principalmente ao desequilíbrio entre a síntese e a degradação protéica, ${ }^{22}$ em que a taxa de degradação protéica é acentuada em relação à síntese, ocorrendo ativação de proteases e, consequentemente, potencialização da proteólise, em parte devido ao sedentarismo, muito comum em idosos..$^{10}$ Com o envelhecimento, o processo de regeneração muscular se torna mais difícil, na medida em que a produção de células estaminais endógenas fica ineficaz, ocorrendo substituição muscular funcional por tecido adiposo e fibroso, com diminuição do compartimento hematopoiético da medula óssea. ${ }^{23} \mathrm{O}$ processo sarcopênico torna-se mais acentuado com o avançar da idade, pois a capacidade de reinervação muscular também diminui. ${ }^{24}$
A perda músculo-esquelética e consequente decréscimo da qualidade e do desempenho músculo-esquelético podem levar à síndrome de fragilidade em idades avançadas, a qual, além de incapacidade e dependência, inclui outros indicadores como perda de peso recente, especialmente da massa magra, autorrelato de fadiga, quedas frequentes, fraqueza muscular, hospitalizações e morte. ${ }^{25}$ Adicionalmente à perda músculo-esquelética, um dos fatores envolvidos na sarcopenia diz respeito às alterações que o sistema neuromuscular sofre com o envelhecimento, como a perda de neurônios motores alfa da medula espinhal, com a subsequente degeneração de seus axônios, ocasionando redução no recrutamento das unidades motoras e, consequentemente, limitação no funcionamento do sistema neuromuscular e aumento da dependência funcional. ${ }^{26}$

$\mathrm{Na}$ habilidade para desempenhar atividades da vida diária (AVDs), a massa e força muscular são fatores relacionados entre si. Pesquisadores demonstraram que mulheres e homens sarcopênicos têm, respectivamente, 3,6 e 4,1 maiores chances de incapacidade, quando comparados àqueles com maior massa muscular. ${ }^{27}$ Da mesma forma, no mesmo estudo, o uso de órteses e maior frequência de quedas foram mais relatados pelos idosos com menor massa muscular. Anteriormente, o mesmo grupo descreveu maior prevalência de sarcopenia em mulheres idosas com maior prejuízo funcional. O estudo ainda mostrou a importância de identificar indivíduos obesos e sarcopênicos, uma vez que o aumento de gordura corporal e a estabilidade do peso dificilmente suscitam interesse sobre a perda muscular. Nesta população, tanto a obesidade quanto a sarcopenia se associaram a limitações funcionais, incapacidade e maior número de quedas, particularmente no sexo feminino. ${ }^{6}$ Esse conjunto de evidências sugere que os mecanismos que levam à sarcopenia são multifatoriais e estão provavelmente interligados (sedentarismo, composição corporal, genética, metabolismo, dieta, etc.), mas ainda precisam ser elucidados (figura 2).

O sedentarismo, sobretudo, é um fator importante para a sarcopenia relacionada ao 
envelhecimento. ${ }^{28}$ Idosos com menor atividade física têm também menor massa muscular e maior prevalência de incapacidade física, 7,10,28 sendo a sarcopenia em idosos associada com uma probabilidade três a quatro vezes maior de incapacidade. $^{6}$ Por outro lado, a prática regular de exercícios desde jovem lentifica a perda muscular do idoso. ${ }^{29} \mathrm{~A}$ intervenção mais eficaz para prevenção e recuperação da perda muscular são os exercícios de resistência. ${ }^{30} \mathrm{Como}$ o envelhecimento é associado a uma perda preferencial de massa muscular em membros inferiores $^{11}$ e o declínio da massa muscular apendicular é mais grave até os 85 anos de idade, ${ }^{4}$ intervenções deveriam ser desenvolvidas levando em conta esses aspectos. Adicionalmente, quando se considera a forte influência da massa muscular esquelética na densidade mineral óssea em mulheres, ${ }^{31}$ para elas em particular o treino de resistência deve ser acompanhado de atividades que envolvam a descarga de peso. ${ }^{11}$

\section{Epidemiologia da sarcopenia}

Os dados sobre a incidência e prevalência da sarcopenia ainda são escassos na literatura do Brasil. Não obstante, em outros países, estudos mostram que a prevalência da sarcopenia varia de acordo com a idade, acometendo de $13 \%$ a $24 \%$ dos indivíduos entre 65 e 70 anos de idade, e mais de $50 \%$ dos idosos acima de 80 anos. ${ }^{4,6,10,11,20,30}$

As variações entre as diferentes prevalências encontradas nos diferentes estudos podem ser devido a diversos fatores, entre os quais diferenças na composição étnica da amostra ${ }^{4,6,10,20}$ e utilização de diferentes parâmetros para a definição de sarcopenia e/ou de métodos de mensuração da massa magra diferentes, tais como densitometria (dual energy X-ray absorptiometry - DXA) ${ }^{4,20} \mathrm{e}$ bioimpedância. ${ }^{6,10}$ Em relação a diferenças entre medidas feitas a partir da massa magra corporal total ou a partir de dados obtidos com a mensuração apenas da massa muscular apendicular (braço e/ ou coxa), parece haver correlação entre essas duas formas de mensuração, tornando possível a diminuição de custos através das medidas de parte dos segmentos corporais. ${ }^{11}$
Apesar das variações nos números da prevalência, é certo que tanto a incidência, quanto a prevalência da sarcopenia aumentam em homens e mulheres com o envelhecimento, mas a partir dos 75 anos de idade a prevalência passa a ser mais elevada em homens (58\%) do que em mulheres (45\%). Isso acontece porque os homens têm maior perda muscular decorrente do declínio do hormônio do crescimento $(\mathrm{GH})$, do fator de crescimento relacionado à insulina (IGF-1) e da testosterona. ${ }^{32-34}$ Contudo, como os homens chegam a essa idade com mais massa magra que as mulheres, sob este aspecto eles guardam maior vantagem sobre as mulheres. ${ }^{11}$

\section{Etiopatogenia da sarcopenia: o papel do} estresse oxidativo e da inflamação

Como se pode observar, o processo de perda de massa e funcionalidade muscular ao longo envelhecimento é um evento inevitável, lento, progressivo e sujeito a diversos fatores intrínsecos e extrínsecos, que estão envolvidos na etiopatogenia da sarcopenia (figura 2).

Recentemente, o entendimento a respeito da etiopatogenia da sarcopenia tem avançado para a biologia celular, com o foco voltado para o metabolismo oxidativo celular. Isto porque alguns autores demonstraram que indivíduos sarcopênicos compartilham os mesmos fatores de risco cardiometabólicos que obesos, diabéticos ou portadores de síndrome metabólica, e que é fundamental avaliar nesses indivíduos, além das AVDs, o perfil lipídico e glicêmico, citocinas inflamatórias e metabolismo oxidativo. ${ }^{35,36}$ No caso, o estresse oxidativo, a disfunção mitocondrial e a inflamação crônica já estão se consolidando na literatura como fatores de risco cardiometabólicos, que por sua vez também desempenham papéis importantes na atrofia muscular relacionada à idade. , $37,38^{3}$

As investigações na área do metabolismo oxidativo têm oferecido suporte considerável para um papel importante das espécies reativas de oxigênio (ERO) na mediação do processo de perda de massa e função muscular associado à 
idade, uma vez que vários estudos demonstram produção elevada de ERO no músculo esquelético durante o envelhecimento. $\mathrm{Na}$ fisiologia normal, a geração de ERO está estreitamente relacionada a algumas respostas adaptativas do músculo para a atividade contrátil, mas durante o envelhecimento essas respostas são severamente prejudicadas. ${ }^{39}$ A interação desses fatores pode convergir em várias vias de sinalização intracelular, afetando o equilíbrio entre a síntese proteica e a degradação, induzindo a apoptose, que pode levar a perda significativa de massa muscular e maior fragilidade e incapacidades (figura 2). ${ }^{9,35}$

Nos organismos aeróbios, a maior parte do oxigênio é reduzida à água dentro da mitocôndria, através da enzima citocromo oxidase, que transfere quatro elétrons ao oxigênio. Entretanto, em decorrência da sua configuração eletrônica (triplet ${ }^{3} \mathrm{O}_{2}$ ), cerca de $2-5 \%$ do oxigênio que se consome tem forte tendência a receber um elétron de cada vez, ou seja, a transferência de elétrons pode ser realizada em passos monoeletrônicos, com a formação de espécies intermediárias. ${ }^{40}$ Deste modo, aproximadamente $2-5 \%$ de todo o oxigênio consumido é convertido em ânion superóxido $\left(\mathrm{O}_{2}^{-}\right)$, que é um radical livre (RL).

Os radicais livres são definidos como um átomo, grupo de átomos ou moléculas com um elétron desemparelhado ocupando um orbital mais externo, sendo, por convenção, representados por $\mathrm{x}^{\bullet}$. Essa definição inclui íons de metais de transição, o átomo de hidrogênio, o óxido nítrico e o dióxido de nitrogênio. ${ }^{40}$ Os radicais livres são conhecidos pelos químicos orgânicos há muitas décadas, porém só recentemente tiveram avaliada sua importância em bioquímica e medicina, pois estão envolvidos em processos como a toxicidade do oxigênio, reações inflamatórias, injúria por reperfusão, câncer e envelhecimento. ${ }^{40-42}$

A toxicidade do oxigênio não se deve apenas aos RL dele derivados, mas também a outros estados do oxigênio, como o radical hidroxila $(\mathrm{OH})$, o oxigênio singlet $\left({ }^{1} \mathrm{O}_{2}\right)$, o óxido nítrico $(\mathrm{ON})$, radicais peróxidos $(\mathrm{ROOH})$, peróxidos nitritos (ONOO-), ácido hipoclórico $\left(\mathrm{HOCl}^{-}\right)$ e peróxido de hidrogênio $\left(\mathrm{H}_{2} \mathrm{O}_{2}\right)$ - este último também é considerado uma ERO, apesar de não possuir um elétron desemparelhado na última órbita. ${ }^{40}$ Dentre as EROs, o peróxido de hidrogênio $\left(\mathrm{H}_{2} \mathrm{O}_{2}\right)$ é a mais estável e a menos reativa. A hidroxila $(\mathrm{OH})$ é a ERO mais reativa, menos estável e mais perigosa do ponto de vista biológico, pois reage imediatamente após sua formação com qualquer molécula biológica ao seu redor. Além disso, diferentemente do peróxido de hidrogênio e do ânion superóxido, não existem defesas enzimáticas contra este radical. Os principais alvos das EROs nas células são os lípides, as proteínas contendo grupamento sulfidril e o ácido desoxirribonucléico (DNA). ${ }^{40}$

Nesse contexto, o estresse oxidativo é resultado do desequilíbrio no status redox da célula, devido ao aumento das concentrações de ERO ou à diminuição na atividade de um ou mais sistemas antioxidantes - ou seja, sob determinadas condições patológicas, a taxa de produção de ERO é maior que a resposta antioxidante, alterando o equilíbrio oxidanteantioxidante. ${ }^{40}$ Estudos têm demonstrado que, com o envelhecimento, os níveis de estresse oxidativo aumentam no músculo esquelético, tanto em repouso, quanto durante a atrofia por desuso, sugerindo que o estresse oxidativo tem papel na mediação induzida pelo desuso muscular e perda de fibras musculares associados à sarcopenia. ${ }^{43-45}$

O estresse oxidativo parece ocupar um espaço subjacente na patogênese de diferentes doenças crônicas (diabetes, hipertensão, câncer, etc) ${ }^{41}$ e inclusive naquelas que são acompanhadas de perda de massa muscular. ${ }^{44}$ Por exemplo, Samba et al. demonstraram que baixos níveis de antioxidantes no plasma estãoindependentemente associados com a diminuição da força muscular esquelética e com deficiência de mobilidade. ${ }^{36}$ Em paralelo, Howard et al. sugerem que o aumento dos níveis de inflamação crônica de baixa intensidade induzida por estresse oxidativo tem demonstrado ser prejudicial para o músculo esquelético em humanos, bem como em modelos animais. ${ }^{45}$ No caso, o processo inflamatório é considerado como um mecanismo subjacente ao envelhecimento e às doenças associadas à 
idade, e pode servir como uma ponte entre o envelhecimento normal e processos patológicos relacionados ao envelhecimento. ${ }^{46,47}$ Além disso, as EROs também parecem funcionar como segundos mensageiros do fator de necrose tumoral- alfa (TNF- $\alpha$ ) no músculo esquelético, ativando o fator de transcrição- $\mathrm{kB}$ (NF-kB), direta ou indiretamente. ${ }^{17}$

$\mathrm{Na}$ verdade, o aumento do estresse oxidativo e inflamação são bem conhecidos e caminham lado a lado em muitas doenças músucloesqueléticas. A inflamação crônica subclínica pode ser um marcador de limitação funcional em idosos em diversas doenças e condições de saúde. ${ }^{48}$ Sobretudo, a patogênese da sarcopenia vem sendo atribuída ao estresse oxidativo, inflamação, alterações endócrinas, inatividade e desnutrição (figura 2). Além disso, muitos dos fatores que têm sido implicados na sarcopenia parecem não agir de forma isolada, e muitas das inter-relações passam pelo estresse oxidativo, que parece desempenhar papel-chave no seu desencadeamento, funcionando como fator primário causal. ${ }^{49}$ Nesse sentido, é importante ressaltar que o acúmulo de danos mitocondriais e do DNA nuclear provocados pelo estresse oxidativo associado ao processo inflamatório levam à perda de miócitos. A disfunção mitocondrial associada à idade está relacionada, não somente com a perda da capacidade de gerar ATP, mas também com a ativação de rotas que levam a perda celular que é característica de processos neurodegenerativos e da própria sarcopenia. ${ }^{50}$

No caso da sarcopenia, a diminuição da capacidade de gerar ATP apresenta-se mais evidente, uma vez que a característica dessa doença é a perda de fibras do tipo IIA, que dependem principalmente da fosforilação oxidativa para a produção de ATP. Essas fibras possuem mais mitocôndrias e vasos sanguíneos para conduzir o oxigênio até as células musculares do que as fibras somente glicolíticas tipo IIB. ${ }^{17}$ A fosforilação oxidativa ocorre na membrana interna das mitocôndrias e necessita de glicose (piruvato) e oxigênio para atuar como aceptor final de elétrons e de íons hidrogênio $\left(\mathrm{H}^{+}\right)$para formar, em última instância, energia e uma molécula de água. A energia liberada pelo transporte de elétrons é utilizada para bombear íons hidrogênio da matriz interna da mitocôndria para a externa, gerando um potencial elétrico que determina o fluxo de íons hidrogênio para o interior da matriz através de uma enzima denominada ATPase. A energia derivada deste fluxo de hidrogênio é então utilizada pela ATPase para converter a molécula de adenosina-difosfato (ADP) em ATP. Quando ocorre aumento na diferença de potencial eletroquímico (gradiente de próton $\left.\left[\mathrm{H}^{+}\right]\right)$produzida na membrana interna da mitocôndria, o tempo de vida das moléculas, que levam a produção do ânion superóxido, também aumenta. ${ }^{17}$ Esse prolongamento na manutenção das moléculas tem como consequência a elevação nos níveis de superóxido. ${ }^{51} \mathrm{O}$ aumento nos níveis de RL provoca danos ao DNA mitocondrial (causando danos à cadeia de transporte de elétrons e disfunção mitocondrial), resultando na redução da recaptação de $\mathrm{Ca}^{2+}$ pelo retículo sarcoplasmático e levando, em última instância, à morte de miofibrilas. ${ }^{12}$

Estudos in vitro têm demonstrado que a atividade contráctil aumenta a produção de ERO no músculo esquelético. $O$ aumento de ERO, principalmente de $\mathrm{H} 2 \mathrm{O} 2$, é menor em comparação com o demonstrado em outros estudos com modelos in vitro em que foram utilizados miotubos obtidos a partir de células do músculo esquelético primário ou de uma linhagem de células do músculo esquelético. ${ }^{52,53}$

No caso das fibras tipo IIB, elas dependem basicamente da glicólise anaeróbia para produzir ATP. Contudo, com a hidrólise do ATP ocorre um acúmulo de $\mathrm{H}^{+}$contribuindo para a formação de ERO e acidose, uma condição implicada no desencadeamento da fadiga muscular. ${ }^{17}$ Deste modo, as fibras do tipo IIB entram em fadiga muito mais rápido que as do tipo IA. ${ }^{17}$ A produção de ERO nas mitocôndrias, que no caso ocorre em ambos os tipos de fibras musculares, pode estar implicada na perda e atrofia das mesmas. Entretanto, alguns autores têm sugerido que as mitocôndrias podem não ser a única fonte dominante de produção de ERO durante a atividade contrátil do músculo esquelético. ${ }^{54}$ 
Alguns estudos indicam que enzimas do complexo NAD (P) H oxidase também podem gerar ERO no retículo sarcoplasmático do músculo cardíaco e esquelético. ${ }^{55}$

Essas enzimas são responsáveis pela geração de superóxido, que parece modular a liberação de cálcio pelo retículo sarcoplasmático através de oxidação de receptores específicos. ${ }^{56}$ Túbulos transversais do músculo esquelético também contêm NAD (P) H oxidase, que igualmente parece estar envolvida com a produção de superóxido para o citosol das células do músculo esquelético. ${ }^{57}$ Essas evidências indicam que existem outras fontes de geração de ERO, além da mitocôndria, como por exemplo, o complexo NAD (P) oxidase e xantina oxidase (também encontradas em células endoteliais), e que acima de tudo essa geração, durante a atividade contráctil músculo-esquelética, desempenha papel fundamental na modulação da função muscular. ${ }^{58}$

Estudo em modelo experimental knockouts para o gene da superoxido dismutase dependente de cobre/zinco (SOD Cu-Zn) mostrou que a ausência desse gene promove a atrofia e acelera a perda de massa e função muscular, sugerindo o papel importante da maquinaria antioxidante no controle da geração de ERO no músculo esquelético. ${ }^{59}$

\section{FATORES EXÓGENOS}

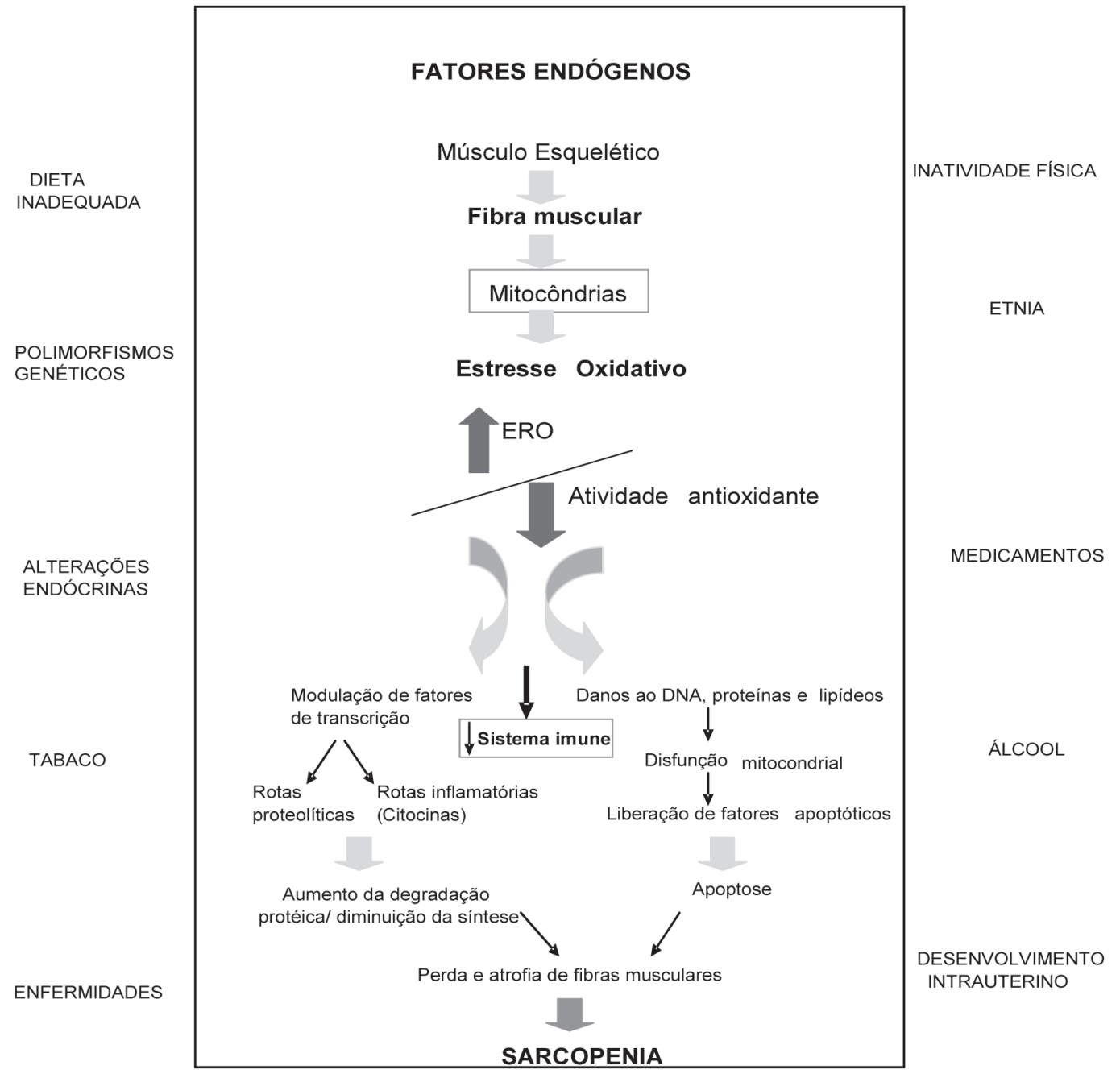

Figura 2. Esquema de incidência e interação de fatores exógenos e endógenos implicados na produção de Espécie Reativa de Oxigênio (ERO) no músculo esquelético, associado ao desenvolvimento da sarcopenia.Fonte: Si-Jin et al.2010. ${ }^{49}$ 
Umachado relevante no estudo da bioenergética e fisiologia mitocondrial e que pode ter implicações na etiologia da sarcopenia foi o encontrado por Silva et. al. ${ }^{60}$ Nesse estudo, conduzido em modelo experimental (camundongos), os autores verificaram que a mitocôndria produz mais ERO quando o organismo está em repouso, respirando em ritmo lento e consumindo pouco oxigênio. Quando a mitocôndria está trabalhando ativamente, mais oxigênio é consumido e menos EROs são gerados. ${ }^{60}$ Esse achado corrobora a evidência de que o sedentarismo é um fator de risco importante para a perda de massa muscular via geração de ERO.

Além dos fatores já apresentados e discutidos, a dieta também desempenha papel fundamental no desencadeamento da sarcopenia, sob o prisma do metabolismo oxidativo, uma vez que a maior fonte de $\mathrm{H}^{+}$(gorduras e carboidratos) e moléculas com potencial antioxidante (vitamina C, E, polifenóis, etc.) são provenientes da dieta. Dessa forma, uma dieta adequada (in natura ou na forma de suplementos) em aporte antioxidante pode ser uma aliada muito importante no controle da produção excessiva de ERO.

Estudo conduzido com modelo experimental (camundongos idosos) mostrou que tanto suplementação dietética com ácido linoleico (a uma concentração de $0.5 \%$ ), quanto a alimentação enriquecida com óleo de milho (concentração de $6 \%)$ por um período de seis meses, mostrou-se eficaz no aumento da produção de ATP e elevada produção muscular de enzimas antioxidantes, como catalase e glutationa peroxidase. Entretanto, somente o grupo de camundongos que recebeu a suplementação ácido linoleico apresentou diminuição de marcadores inflamatórios (IL6 e TNF- $\alpha$ ) e oxidativo (malondialdeído). Esse achado sugere que a suplementação com ácido linoleico pode modular o estresse oxidativo e inflamação e pode atuar como um coadjuvante na prevenção da sarcopenia durante o processo de envelhecimento. ${ }^{61}$

Outro estudo conduzido com ratos adultos e idosos divididos em grupos que recebiam uma suplementação de uma mistura de antioxidantes (rutina, vitamina E, vitamina A, zinco e selênio) ou não (placebo), para avaliar a capacidade da leucina estimular a síntese proteica, mostrou que, depois da suplementação, a concentração de leucina aumentou. Em ratos idosos, a capacidade de a leucina estimular a síntese proteica muscular é menor do que em ratos adultos. Contudo, esse efeito foi revertido quando ratos idosos foram suplementados com a mistura de antioxidantes. Os resultados desse estudo sugerem que a suplementação com antioxidantes pode beneficiar o metabolismo proteico muscular durante o envelhecimento, o que pode ter impacto positivo na perda de massa muscular esquelética. ${ }^{62}$

A dieta é fundamental para o aporte de proteínas, que está, por sua vez, associado ao metabolismo proteico do tecido músculoesquelético, ${ }^{63}$ e também com presença de maior ingestão de proteína total, com menor perda de massa muscular. ${ }^{64}$ Em idosos, os aminoácidos essenciais podem estimular a síntese de proteínas no músculo, mas poucos estudos são conduzidos nessa área com humanos idosos.

Em 2003, foi realizado um ensaio clínico com idosos e adultos saudáveis que aliou a suplementação de 1000IU/d de vitamina E e exercício físico ou placebo por 12 semanas. O estudo foi delineado da seguinte forma: 16 jovens com idade média de 26 anos e 16 idosos com idade média de 71 anos (todos homens e saudáveis) foram aleatoriamente randomizados em dois grupos: um consumiu 1000 UI de vitamina $\mathrm{E} / \mathrm{d}$ e realizava o exercício excêntrico e o outro era placebo; ambos foram acompanhados durante 12 semanas. Antes e após a ingestão de vitamina $\mathrm{E}$, o grupo não-placebo realizava uma corrida (lomba abaixo) por 45 min a 75\% VO (2) máxima. As amostras de sangue eram coletadas antes e imediatamente pós-exercício $(0 \mathrm{~h})$, e em 6 , 24 e 72 h pós-exercício, para determinar o status antioxidante, lesão muscular, peroxidação lipídica e danos ao DNA. Os resultados mostraram que, após o exercício, os homens jovens e os idosos apresentaram aumentos similares na creatina quinase sérica (CK), F (2alpha)-isoprostanos (iPF (2alpha) e malondialdeído. A vitamina $\mathrm{E}$ diminuiu o pico CK em homens jovens, enquanto que nos homens idosos diminuiu os níveis de 
repouso da iPF (2alpha) e suprimiu o aumento de iPF (2 alfa) depois das $24 \mathrm{~h}$ pós-exercício. Além disso, verificou-se que o dano ao DNA, medido através da 8-hidroxi-2'-desoxiguanosina (8-OHdG) não foi alterado pelo exercício. ${ }^{65}$

Os resultados obtidos com o estudo sugeriram que a suplementação de vitamina E, aliada ao exercício excêntrico, induz modestas alterações no metabolismo oxidativo, embora tenham sido observadas diferenças entre homens jovens e adultos. Ao mesmo tempo, o estudo sugeriu que a idade não apresenta influência direta sobre essas reações no grupo de indivíduos fisicamente aptos. ${ }^{65}$

Mesmo que o estudo não tenha encontrado alterações robustas nas variáveis avaliadas, tanto o exercício físico quanto a suplementação com vitamina $\mathrm{E}$ desempenham função importante na modulação de alguns parâmetros fisiológicos que podem estar envolvidos com o surgimento da sarcopenia. Por isso, um aporte nutricional adequado (qualidade e quantidade dos alimentos), aliado à atividade ou exercício físico, é fundamental para uma metabolização ideal dos nutrientes pelo organismo, com vistas a manutenção e reparo músculo-esquelético. ${ }^{63}$

\section{RESULTADOS}

Com base na revisão da literatura sobre o papel do estresse oxidativo na gênese da sarcopenia, os resultados dos diferentes estudos aqui descritos sugerem que os mecanismos que levam à sarcopenia são multifatoriais e estão provavelmente interligados, e que o desbalanço redox celular e a inflamação desempenham papéis de base na perda de fibras musculares, levando à perda de massa músculo-esquelética e podendo desencadear o processo sarcopênico associado ao envelhecimento. ${ }^{9,37,38}$

Além disso, os diferentes estudos citados demonstram que o estilo de vida, como a dieta e atividade física, também ocupam posição central no aumento do estresse oxidativo e surgimento da sarcopenia, via ingestão de substâncias antioxidantes, inserir proteínas e gasto energético (quanto mais atividade física, mais oxigênio é consumido e menos ERO são produzidas), respectivamente. ${ }^{36,60,62} \mathrm{E}$, ainda, que a etiologia da sarcopenia vem sendo atribuída ao estresse oxidativo, inflamação, alterações endócrinas (hormônios sexuais), inatividade e má nutrição.

Contudo, muitos fatores associados à sarcopenia parecem não agir de forma isolada, e muitas das inter-relações passam pelo estresse oxidativo e indução da inflamação, funcionando como fator primário causal na perda e atrofia de fibras musculares. $9,43,49$

\section{CONSIDERAÇÕES FINAIS}

Ainda existe muita controvérsia em se classificar a sarcopenia como uma doença, porque a perda de massa músculo-esquelética é considerada um fenômeno inerente ao processo de envelhecimento. Entretanto, quando a perda é acentuada, provocando dependência funcional e interferindo de forma negativa na qualidade de vida dos idosos, dever-se-ia classificá-la como doença.

A etiologia da sarcopenia é multifatorial e envolve a interação de diversos fatores, incluindo aspectos genéticos, metabólicos e de estilo de vida, como os hábitos alimentares e de gasto energético (atividade física). Sobretudo recentemente, a literatura tem reportado que a etiopatogenia de diversas DCNTs está intimamente relacionada com a quebra da homeostase do metabolismo oxidativo, promovendo aumento na taxa de produção de ERO, causando danos mitocondriais, alterando o sistema imunológico e inflamatório e, consequentemente, afetando o sistema orgânico como um todo. O acúmulo de danos causados por estas substâncias às biomoléculas ao longo da vida reduz a capacidade fisiológica da maioria dos sistemas, como o cardiovascular, o respiratório, o neuromuscular e o metabólico. Por conseguinte, esses danos tornam o indivíduo mais susceptível às doenças crônicas, como as cardiovasculares, as neurodegenerativas, as neoplasias e a própria sarcopenia. ${ }^{41} \mathrm{Em}$ idosos, esse desequilíbrio no metabolismo oxidativo se torna mais perigoso, 
uma vez que, associado ao declínio dos hormônios sexuais, que exercem efeitos anabólicos sobre o tecido músculo-esquelético, pode acelerar a perda e atrofia desse tecido., ${ }^{9,43}$

Dessa forma, é necessário ter uma visão sistêmica dos mecanismos fisiológicos que levam à perda de massa músculo-esquelética ao longo do envelhecimento, bem como da incidência e interação de fatores envolvidos nesse processo para poder preveni-lo e/ou postergá-lo. Sobretudo, é fundamental investir na prevenção dessa doença desde a infância, com enfoque no ganho ideal de massa músculo-esquelética, que deve passar por dieta equilibrada e saudável, atividade física adequada à faixa etária, bem como um entorno socioambiental saudável, para se chegar a idades avançadas com boa reserva fisiológica.

\section{REFERÊNCIAS}

1. Troen RB. The Biology of Aging. Mt Sinai J Med 2003; 70(1):3-22.

2. Goodpaster BH, Park SW, Harris TB, Kritchevsky SB, Nevitt M, Schwartz AV, et al. The loss of skeletal muscle strength, mass, and quality in older adults: The health, aging and body composition study. J Gerontol A Biol Sci Med Sci . 2006 Oct;61(10):1059-64.

3. Rosenberg IH. Sarcopenia: origins and clinical relevance. J Nutr 1997; 127: 990S-1S.

4. Gillette-Guyonnet S, Nourhashemi F, Andrieu S, Cantet C, Albarède JL, Vellas B, et al. Body Composition in French women 75+ years of age: the EPIDOS study. Mech Aging Dev. 2003 Mar;124 (3):311-6.

5. Baumgartner RN, Waters DL, Gallagher D, Morley JE, Garry PJ. Predictors of skeletal muscle mass in elderly men and women. Mech Ageing Dev.1999 Mar 1;107(2):123-36.

6. Baumgartner RN, Koehler KM, Gallagher D, Romero L, Heymsfield SB, Ross RR, et al. Epidemiology of sarcopenia among the elderly in New Mexico. Am J Epidemiol. 1998 Apr 15;147(8):755-63.

7. Landi F, Liperoti R, Fusco D, Mastropaolo S, Quattrociocchi D, Proia A, et al. Prevalence and Risk Factors of Sarcopenia Among Nursing Home
Além disso, é premente direcionar as pesquisas para um foco interdisciplinar, integrando os campos das ciências biológicas, nutrição, fisioterapia, educação física e das diferentes áreas da medicina, para se começar a montar o grande quebra-cabeça que envolve a etiologia da sarcopenia.

\section{AGRADECIMENTOS}

Agradecemos especialmente ao Programa Nacional de Pós-Doutorado da Coordenação de Aperfeiçoamento de Pessoal de Nível SuperiorCAPES, pela bolsa e auxílio financeiro na realização deste trabalho, e à Profa. Dra. Carla Helena Augustin Schwanke, do Programa de Pós-Graduação em Gerontologia Biomédica da PUC-RS, pela cedência de material bibliográfico para a elaboração deste manuscrito.

Older Residents. J Gerontol A Biol Sci Med Sci. 2012 Jan; 67(1):48-55.

8. Deschenes MR. Effects of aging on muscle fibre type and size. Sports Med. 2004; 34(12):809-24.

9. Silva TAA, Frisoli Junior A, Pinheiro MM, Szejnfeld VL. Sarcopenia associada ao envelhecimento: aspectos etiológicos e opções terapêuticas. Revista Brasileira de Reumatologia 2006;46(6):391-7.

10. Castillo EM, Goodman-Gruen D, Kritz-Silverstein D, Morton DJ, Wingard DL, Barrett-Connor E. Sarcopenia in Elderly Men and Women The Rancho Bernardo Study. Am J Prev Med. 2003 Oct; 25(3): 226-31.

11. Janssen I, Baumgartner RN, Ross R, Rosenberg IH, Roubenoff R. Skeletal Muscle Cutpoints Associated with Elevated Physical Disability Risk in Older Men and Women. Am J Epidemiol. 2004 Feb 15; 159(4):413-21.

12. Fulle S, Protasi F, Di Tano G, Pietrangelo T, Beltramin A, Boncompagni S, et al. The contribution of reactive oxygen species to sarcopenia and muscle ageing. Exp Gerontol. 2004 Jan; 39(1):17-24.

13. Jang YC, Lustgarten MS, Liu Y, Muller FL, Bhattacharya $\mathrm{A}$, Liang $\mathrm{H}$, et al. Increased superoxide in vivo accelerates age-associated muscle atrophy through mitochondrial dysfunction and 
neuromuscular junction degeneration. FASEB J. 2010 May;24(5):1376-90.

14. Alemán H, Esparza J, Ramirez FA, Astiazaran H, Payette $\mathrm{H}$. Longitudinal evidence on the association between interleukin- 6 and $\mathrm{C}$-reactive protein with the loss of total appendicular skeletal muscle in freeliving older men and women. Age and Ageing 2011 Jul;40(4):469-75.

15. Mecocci P, Fanó G, Fulle S, MacGarvey U, Shinobu L, Polidori MC, et al. Age-dependent increases in oxidative damage to DNA, lipids, and proteins in human skeletal muscle. Free Radic Biol Med. 1999 Feb;26(3-4):303-8.

16. Morley JE, Baumgartner RN, Roubenoff R, Mayer J, Nair KS. Sarcopenia. J Lab Clin Med. 2001 Apr;137(4):231-43.

17. Silverthorn DU. Músculos. In: Fisiologia humana: uma abordagem integrada. 5. ed. Porto Alegre: Artmed; 2010

18. Matsudo SSM, Matsudo VKR, Barros Neto TL. Impacto do envelhecimento nas variáveis antropométricas, neuromotoras e metabólicas da aptidão física. Revista brasileira de ciência \& movimento. 2000;08(04): 21-32.

19. Frontera WR, Hughes VA, Lutz KJ, Evans WJ. A cross sectional study of muscle strength and mass in 45- to 78-yr-old men and women. J Appl Phys. 1991 Aug; 71(2): 644-50.

20. Lau EM, Lynn HS, Woo JW, Kwok TC, Melton LJ 3rd. Prevalence of and risk factors for sarcopenia in elderly Chinese men and women. J Gerontol A Biol Sci Med Sci. 2005 Feb; 60(2):213-6.

21. Lang T, Streeper T, Cawthon P, Baldwin K, Taaffe DR, Harris TB, et al. Sarcopenia: etiology, clinical consequences, intervention, and assessment. Osteoporos Int. 2010 Apr;21(4):543-59.

22. Cruz-Jentoft AJ, Baeyens JP, Bauer JM, Boirie Y, Cederholm T, Landi F, et al. Sarcopenia: European consensus on definition and diagnosis. Report of the European Working Group on Sarcopenia in Older People. Age Ageing. 2010 Jul;39(4):412-23.

23. Visser M, Kritchevsky SB, Goodpaster BH, Newman AB, Nevitt M, Stamm E, et al. Leg muscle mass and composition in relation to lower extremity performance in men and women aged 70 to 79 : the health, aging and body composition study. J Am Geriatr Soc. 2002 May; 50(5):897-904.

24. Silva H, Conboy IM. Aging and Stem Cell Renewal. StemBook [Internet]. Cambridge (MA): Harvard Stem Cell Institute; 2008-2008 Jul 15. Disponível em: www.ncbi.nlm.nih.gov/bookshelf/br.fcgi?book =stembook\&part=agingandstemcellrenewal.
25. Roubenoff R, Hughes VA. Sarcopenia: current concepts. J Gerontol A Biol Sci Med Sci. 2000 Dec;55(12):M716-24.

26. Roubenoff, R. Origins and clinical relevance of sarcopenia. Can J Appl Physiol. 2001 Feb;26(1):78-89.

27. Baumgartner RN. Body composition in healthy aging. Ann N Y Acad Sci. 2000 May; 904:437-48.

28. Evans WJ. Effects of exercise on senescent muscle. Clin Orthop Relat Res. 2002 Oct;(403 Suppl):S211-20.

29. Freiberger E, Sieber C, Pfeifer K. Physical activity, exercise, and sarcopenia - future challenges. Wien Med Wochenschr. 2011 Sep;161(17-18):416-25. DOI 10.1007/s10354-011-0001-z.

30. Janssen I, Shepard I, Katzmarzyk DS, Roubenoff $\mathrm{R}$. The healthcare costs of sarcopenia in the United States. J Am Geriatr Soc. 2004 Jan;52(1):80-5.

31. Snow-Harter C, Bouxsein M, Lewis B, Charette S, Weinstein P, Marcus R. Muscle strength as a predictor of boné mineral density in young women. J Bone Miner Res. 1990; 5(6): 589-95.

32. Giovannini S, Marzetti E, Borst SE, Leeuwenburgh C. et al. Modulation of GH/IGF-1 axis: potential strategies to counteract sarcopenia in older adults. Mech Ageing Dev. 2008 Oct;129(10):593-601.

33. Ceda GP, Dall'Aglio E, Maggio M, Lauretani F, Bandinelli S, Falzoi C, et al. Clinical implications of the reduced activity of the GH-IGF-I axis in older men. J Endocrinol Invest 2005;28(11 Suppl Proceedings):96-100.

34. Kamel HK, Maas D, Duthie EH Jr. Role of hormones in the pathogenesis and management of sarcopenia. Drugs Aging. 2002;19(11):865-77.

35. Sanada K, Sanada K, Miyachi M, Tanimoto M, Yamamoto K, Murakami H, Okumura S, et al. A cross-sectional study of sarcopenia in Japanese men and women: reference values and association with cardiovascular risk factors. Eur J Appl Physiol. 2010;110(1):57-65.

36. Samba RD, Lauretani F, Ferrucci L. Carotenoids as protection against sarcopenia in older adults. Arch Biochem Biophys. 2007 Feb 15;458(2):141-5.

37. Carter CS, Carter CS, Hofer T, Seo AY, Leeuwenburgh C, et al. Molecular mechanisms of life- and healthspan extension: Role of calorie restriction and exercise intervention. Appl Physiol Nutr Metab. 2007 Oct;32(5): 954-66.

38. Valle Gottlieb MG, Cruz IB, Duarte MM, Moresco $\mathrm{RN}$, Wiehe M, Schwanke CH et al. Association among metabolic syndrome, ischemia, inflamatory, oxidatives and lipids biomarkers. J Clin Endocrinol Metab. 2010 Feb;95(2):586-91. 
39. Jackson MJ. Reactive oxygen species and redoxregulation of skeletal muscle adaptations to exercise. Philos Trans R Soc Lond B Biol Sci . 2005 Dec 29;360(1464):2285-91.

40. Dröge W. Free Radicals in the Physiological Control of Cell Function. Physiol Rev. 2002 Jan;82(1):47-95.

41. Matés JM, Pérez-Gomez C, Castro IN. Antioxidant enzymes and human diseases. Clin Biochem 1999 Nov;32(8):595-603.

42. Cerutti PA. Oxy-radicals and cancer. Lancet 1994 Sep 24; 344 (8926):862-3.

43. Siu PM, Pistilli EE, Alway SE. Age-dependent increase in oxidative stress in gastrocnemius muscle with unloading. J Appl Physiol 2008 Dec;105(6):1695-705.

44. Moylan JS, Reid MB. Oxidative stress, chronic disease, and muscle wasting. Muscle Nerve. 2007 Apr;35(4):411-29.

45. Howard C, Ferrucci L, Sun K, Fried LP, Walston $\mathrm{J}$, Varadhan R, et al. Oxidative protein damage is associated with poor grip strength among older women living in the community. J Appl Physiol. 2007 Jul;103(1):17-20.

46. Reid MB, Li YP. Tumor necrosis factor-alfa and muscle wasting: A cellular perspective. Respir Res. 2001; 2(5):269-72.

47. Chung HY, Cesari M, Anton S, Marzetti E, Giovannini S, Seo AY, et al. Molecular inflammation: Underpinnings of aging and agerelated diseases. Ageing Res Rev. 2009 Jan;8(1):18-30.

48. Brinkley TE, Leng X, Miller ME, Kitzman DW, Pahor M, Berry MJ, et al. Chronic inflammation is associated with low physical function in older adults across multiple comorbidities. J Gerontol A Biol Sci Med Sci. 2009 Apr;64 (4):455-61.

49. Si-Jin M, Long-Jiang Y. Oxidative Stress, Molecular Inflammation and Sarcopenia. Int J Mol Sci. 2010 Apr 12;11(4):1509-26.

50. Figueiredo PA, Mota MP, Appell HJ, Duarte JA. The role of mitochondria in aging of skeletal muscle. Biogerontology. 2008 Apr;9(2):67-84.

51. Korshunov SS, Skulachev VP, Starkov AA. High protonic potencial actuates a mechanism of production of reative oxygen species in mitochondria. FEBS Lett.1997; 416: 15-8.

52. McArdle F, Pattwell DM, Vasilaki A, McArdle A, Jackson MJ, et al. Intracellular generation of reactive oxygen species by contracting skeletal muscle cells. Free Radic Biol Med. 2005 Sep 1;39(5):651-7.

53. Vasilaki A, Csete M, Pye D, Lee S, Palomero J, McArdle F, et al. Genetic modification of the manganese superoxide dismutase/glutathione peroxidase1 pathway influences intracellular ROS generation in quiescent, but not contracting, skeletal muscle cells. Free Radic Biol Med. 2006;41(11):1719-25.

54. Jackson MJ, Pye D, Palomero J. The production of reactive oxygen and nitrogen species by skeletal muscle. J Appl Physiol. 2007 Apr;102(4):1664-70.

55. Xia R, Xia R, Webb JA, Gnall LL, Cutler K, Abramson JJ, et al. Skeletal muscle sarcoplasmic reticulum contains a NADH-dependent oxidase that generates superoxide. Am J Physiol Cell Physiol 2003; 285:C215-C21.

56. Cherednichenko G, Zima AV, Feng W, Schaefer S, Blatter LA, Pessah IN et al. NADH oxidase activity of rat cardiac sarcoplasmic reticulum regulates calcium-induced calcium release. Circ Res. 2004;94(4):478-86.

57. Espinosa A, Leiva A, Peña M, Müller M, Debandi A, Hidalgo $\mathrm{C}$, et al. Myotube depolarization generates reactive oxygen species through $\mathrm{NAD}(\mathrm{P}) \mathrm{H}$ oxidase; ROS-elicited Ca2+ stimulates ERK, CREB, early genes. J Cell Physiol. 2006 Nov; 209(2):379-88.

58. Palomero J, Jackson MJ. Redox regulation in skeletal muscle during contractile activity and aging. J Anim Sci. 2010 Apr;88(4):1307-13.

59. Muller FL, Song W, Liu Y, Chaudhuri A, PiekeDahl S, Strong R, et al. Absence of CuZn superoxide dismutase leads to elevated oxidative stress and acceleration of age-dependent skeletal muscle atrophy. Free Radic Biol Med. 2006 Jun 1;40(11):1993-2004

60. Silva WS, Gómez-Puyou A, Gómez-Puyou MT, Moreno-Sanchez R, De Felice FG, Meis L, et al. Mitochondrial Bound Hexokinase: Activity as a Preventive Antioxidant Defense: Steady State ADP Formation as a Regulatory Mechanism of Membrane Potential and Reactive Oxygen Species Generation in Mitochondria. J Biol Chem 2004;279(38):39846-55.

61. Rahman M, Halade GV, El Jamali A, Fernandes G. et al. Conjugated linoleic acid (CLA) prevents ageassociated skeletal muscle loss. Biochem Biophys Res Commun. 2009;383(4):513-8.

62. Marzani B, Balage M, Vénien A, Astruc T, Papet I, Dardevet D, et al. Antioxidant supplementation restores defective leucine stimulation of protein synthesis in skeletal muscle from old rats. J Nutr. 2008;138(11):2205-11.

63. Paddon-Jones $\mathrm{D}$, et al. Role of dietary protein in the sarcopenia of aging. Protein Summit 2007: Exploring the Impact of High-Quality Protein on Optimal Health. Am J Clin Nutr. 2008;87(5):1562S-6S. 
64. Chaput JP, Lord C, Cloutier M, Aubertin Leheudre M, Goulet ED, Rousseau S, et al. Relationship between antioxidant intakes and class I sarcopenia in elderly men and women. J Nutr Health Aging. 2007;11(4):363-9.
65. Sacheck JM, Milbury PE, Cannon JG, Roubenoff $\mathrm{R}$, Blumberg JB. Effect of vitamin e and eccentric exercise on selected biomarkers of oxidative stress in young and elderly men. Free Radic Biol Med. 2003;34(12):1575-88.

Recebido: 30/11/2010

Reapresentado: 05/10/2011

Aprovado: 06/12/2011 